\title{
Atrophy in the parahippocampal gyrus as an early biomarker of Alzheimer's disease
}

\author{
C. Echávarri • P. Aalten · H. B. M. Uylings • \\ H. I. L. Jacobs • P. J. Visser · E. H. B. M. Gronenschild • \\ F. R. J. Verhey $\cdot$ S. Burgmans
}

Received: 26 June 2010/ Accepted: 29 September 2010/Published online: 19 October 2010

(C) The Author(s) 2010. This article is published with open access at Springerlink.com

\begin{abstract}
The main aim of the present study was to compare volume differences in the hippocampus and parahippocampal gyrus as biomarkers of Alzheimer's disease (AD). Based on the previous findings, we hypothesized that there would be significant volume differences between cases of healthy aging, amnestic mild cognitive impairment (aMCI), and mild AD. Furthermore, we hypothesized that there would be larger volume differences in the parahippocampal gyrus than in the hippocampus. In addition, we investigated differences between the anterior, middle, and posterior parts of both structures. We studied three groups of participants: 18 healthy participants without memory decline, 18 patients with aMCI, and 18 patients with mild AD. $3 \mathrm{~T}$ T1-weighted MRI scans were acquired and gray matter volumes of the anterior, middle, and posterior parts of both the hippocampus and parahippocampal gyrus were measured using a manual tracing approach. Volumes of both the hippocampus and parahippocampal gyrus were significantly different between the
\end{abstract}

C. Echávarri $(\bowtie) \cdot$ P. Aalten · H. B. M. Uylings ·

H. I. L. Jacobs - P. J. Visser - E. H. B. M. Gronenschild .

F. R. J. Verhey $\cdot$ S. Burgmans

Department of Psychiatry and Neuropsychology,

School for Mental Health and Neuroscience/Alzheimer Center,

Limburg Maastricht University Medical Center,

6200 MD Maastricht, The Netherlands

e-mail: echavarrizalba@hotmail.com

H. B. M. Uylings

Department of Anatomy and Neuroscience,

VU University Medical Center, Amsterdam,

The Netherlands

P. J. Visser

Alzheimer Center, VU University Medical Center,

Amsterdam, The Netherlands groups in the following order: healthy $>\mathrm{aMCI}>\mathrm{AD}$. Volume differences between the groups were relatively larger in the parahippocampal gyrus than in the hippocampus, in particular, when we compared healthy with aMCI. No substantial differences were found between the anterior, middle, and posterior parts of both structures. Our results suggest that parahippocampal volume discriminates better than hippocampal volume between cases of healthy aging, aMCI, and mild $\mathrm{AD}$, in particular, in the early phase of the disease. The present results stress the importance of parahippocampal atrophy as an early biomarker of AD.

Keywords Alzheimer's disease - Cognitive aging . Mild cognitive impairment · Volumetric magnetic resonance image

\section{Introduction}

Atrophy in the medial temporal lobe has repeatedly been associated with the age-related memory decline, mild cognitive impairment (MCI), and dementia (Burgmans et al. 2009; Hackert et al. 2002; Laakso et al. 2000; Raz et al. 2005; Scher et al. 2007; van de Pol et al. 2006; Visser et al. 2002; Wang et al. 2006). This is, in particular, true for hippocampal atrophy, which is considered to be one of the best predictors of Alzheimer's disease (AD) (Detoledo-Morrell et al. 1997; Jack et al. 1997; 2010; Pennanen et al. 2004). Recent findings suggest, however, that parahippocampal atrophy might have more potential as a predictor of $\mathrm{AD}$.

Previous histological studies have already shown that the earliest neuropathological changes in $\mathrm{AD}$ appear in the entorhinal cortex, which is the anterior part of the parahippocampal gyrus (Braak and Braak 1990; Van Hoesen 
1982). At present, several MRI studies suggest that volume measures of the entorhinal cortex provide higher sensitivity than volume measures of the hippocampus when it comes to detecting $\mathrm{AD}$ in an early phase of the disease (DetoledoMorrell et al. 2004; Dickerson et al. 2001; Pennanen et al. 2004). Despite these findings, the parahippocampal gyrus has until now received much less attention than the hippocampus as an early predictor of AD. Moreover, the total parahippocampal volume-including posterior parts of the gyrus - has rarely been investigated in clinical populations. There is only scant evidence that this posterior part is involved in age-related and pathological changes (Burgmans et al. 2009; Insausti et al. 1998; Thangavel et al. 2008; Weniger and Irle 2006).

There is some evidence that the amount of atrophy differs between anterior, middle, and posterior areas of the hippocampus and parahippocampal gyrus. One hypothesis is that differences in the amount of atrophy along the longitudinal axis are caused by the way cortical connections are organized. Van Hoesen et al. (1982) and Insausti and Amaral (2008) concluded from nonhuman primate studies that the posterior parahippocampal gyrus plays a key role in the information flow between cortical association areas and the hippocampal formation. There are massive projections from the posterior parahippocampal areas $\mathrm{TF}$ and $\mathrm{TH}$ to the rostral portion of the entorhinal cortex. Since the entorhinal cortex is reciprocally connected to the anterior hippocampus, we may speculate that damage in the posterior parahippocampal gyrus can lead to tissue changes in the anterior hippocampus. However, more research is needed to reveal the mechanism of differential decline in the medial temporal lobe structures.

In the present study, we compared volume differences in the hippocampus and parahippocampal gyrus in three groups of older individuals: healthy controls, individuals with amnestic MCI (aMCI), and patients with AD. We hypothesized that volume differences between these groups are larger in the parahippocampal gyrus than in the hippocampus. In addition, we investigated the differences between the anterior, middle, and posterior parts of both structures.

\section{Materials and methods}

\section{Participants}

Three groups of older male participants were included in this study: healthy participants without memory impairment, patients with aMCI, and patients with AD. The healthy participants were recruited by means of advertisements in local newspapers. They were administered an extensive neuropsychological test battery and were included if their performance did not deviate from normal on the verbal learning test (Folstein et al. 1975; Van der Elst et al. 2005). The patients with aMCI or AD were recruited from the Memory Clinic of the Maastricht University Hospital. All the diagnoses were made by a multidisciplinary team under the supervision of an experienced neuropsychiatrist from the Memory Clinic (FRJV) according to the Petersen criteria for aMCI (with at least an impairment in the memory domain) (Petersen et al. 2001; Petersen et al. 1999), and according to the DSM-IV and NINCDS-ADRDA criteria for AD (McKhann et al. 1984). The diagnoses were based on the medical history, co-morbidity, course, and MRI scan. The MRI scan was used to exclude vascular pathology; levels of atrophy were not used for the diagnosis of aMCI and AD.

The exclusion criteria were use of psychoactive medication, abuse of alcohol and drugs, other past or present psychiatric or neurological diseases or serious system diseases, and structural abnormalities in the brain that could account for the cognitive decline. Two participants in the control group were excluded because a brain infarct was detected on the MRI scans and two patients in the AD group were excluded because their MRI images showed motion artefacts. The remaining number of participants (after excluding these four subjects) was 18 per group. Thus, the final population consisted of: 18 healthy male participants (mean age $=64.5$ years, SD age $=3.3$ years); 18 male patients with aMCI (mean age $=65.11$ years, SD age $=4.5$ years), and 18 male patients with $\mathrm{AD}$ (mean age $=72.2$ years, SD age $=9.7$ years). A standardized eight-point scale was used to indicate educational level ( $1=$ primary school, $8=$ university). Written informed consent was obtained from all participants and from the primary caregiver of the AD patients. The study was approved by the local Medical Ethics Committee of the Maastricht University Medical Center.

Image acquisition and analysis

MRI scans were acquired with a 3 T Gyroscan NT MRI scanner (Philips, Best, The Netherlands). Structural T1 images were acquired in the sagittal plane using an MPRAGE sequence $(\mathrm{TR}=8, \mathrm{TE}=3.7 \mathrm{~ms}, \quad \mathrm{FA}=9$, $\mathrm{FOV}=240 \times 240$, matrix size $=240 \times 240$, number of slices $=180 ;$ voxel size $=1 \times 1 \times 1 \mathrm{~mm}^{3}$ ). For data analysis, we used a manual tracing approach. The images were viewed using GIANT (General Image Analysis Tools) (Gronenschild et al. 2010), which is a customized software program that allows tracing of regions of interest in a tri-planar and a rotatable 3D surface-rendered view as well as calculation of volumes of interest. The definitions of boundaries and divisions of the hippocampus and parahippocampal gyrus were performed according to the 
criteria described in a previous publication (Burgmans et al. 2009). Volumetric measures were obtained of the anterior, middle, and posterior parts of the hippocampus and parahippocampal gyrus (Fig. 1).

A single rater (CEZ), who was blind to the demographic and cognitive characteristics of the participants, traced all structures. Intra-rater reliability was determined by the intraclass correlation coefficient (ICC) (Shrout and Fleiss 1979). Ten randomly selected brains were measured twice, and these yielded high test-retest reliability. The ICC of the total hippocampal volume was 0.97 (95\% confidence interval $=0.91,0.99)$, and the ICC of the total parafhippocampal volume was $0.95 \quad(95 \%$ confidence interval $=0.84,0.98)$. To correct for individual differences in unatrophied brain, intracranial volumes were measured using an automated method (FSL Brain Extraction Tool) developed at the Oxford Centre for Functional MRI of the brain (Smith 2002).

\section{Statistical analysis}

Statistical analyses were performed using the Statistical Package for the Social Sciences (SPSS Inc, Chicago) version 16.0 for Windows. To compare the mean age, educational level, and MMSE scores between the three groups, ANOVA (General Linear Model; GLM) was performed. For the volumetric comparisons between the three groups, ANCOVA (GLM) was used, with volume as dependent variable, group as independent variable, and intracranial volume and age as covariates.
Three main things were tested in the ANCOVA analysis. First, the effect of group was calculated to test the overall difference across the three groups. Second, contrasts were calculated by pairwise comparisons among means to test the differences between the two groups: i.e., control versus MCI; control versus AD; and aMCI versus AD. Third, the hypothesis that group differences are larger in the parahippocampal gyrus than in the hippocampus was tested by adding the hippocampus as covariate in the ANCOVA analysis with the parahippocampal gyrus as dependent variable. If group differences are still significant in this analysis, we can state that the parahippocampal gyrus has added value in discriminating between groups on top of the discriminating ability of the hippocampus. For all tests, Pearson's correlation coefficient was calculated as a measure of effect size.

\section{Results}

The three groups differed significantly with respect to age $(p=0.001)$ and MMSE $(p=0.000)$, but did not differ with respect to educational level $(p>0.697)$. The AD group was older (mean age 72.2, SD 9.7) than the other two groups. Minimental scores differed in the following order: control (mean MMSE score 28.8, range 27-30) > MCI (mean MMSE score 27.6, range 22-30) > AD (mean MMSE score 21.0, range 10-28). 16 of the 18 AD patients were in a mild stage of the disease (mean MMSE score 21.0 , range $18-28$ ), and two patients in a moderate-severe
Fig. 1 Patterns in the top panel show the subdivision of the hippocampus and parahippocampal gyrus along the antero-posterior axis. The anterior part included the anterior $35 \%$ of the coronal slices (and any rounded off number of slices closest to this cutoff), the middle part comprised $35 \%$, and the posterior part included the remaining $30 \%$. The bottom panel shows the coronal sections of the hippocampus (red) and parahippocampal gyrus (blue) along the anteriorposterior axis
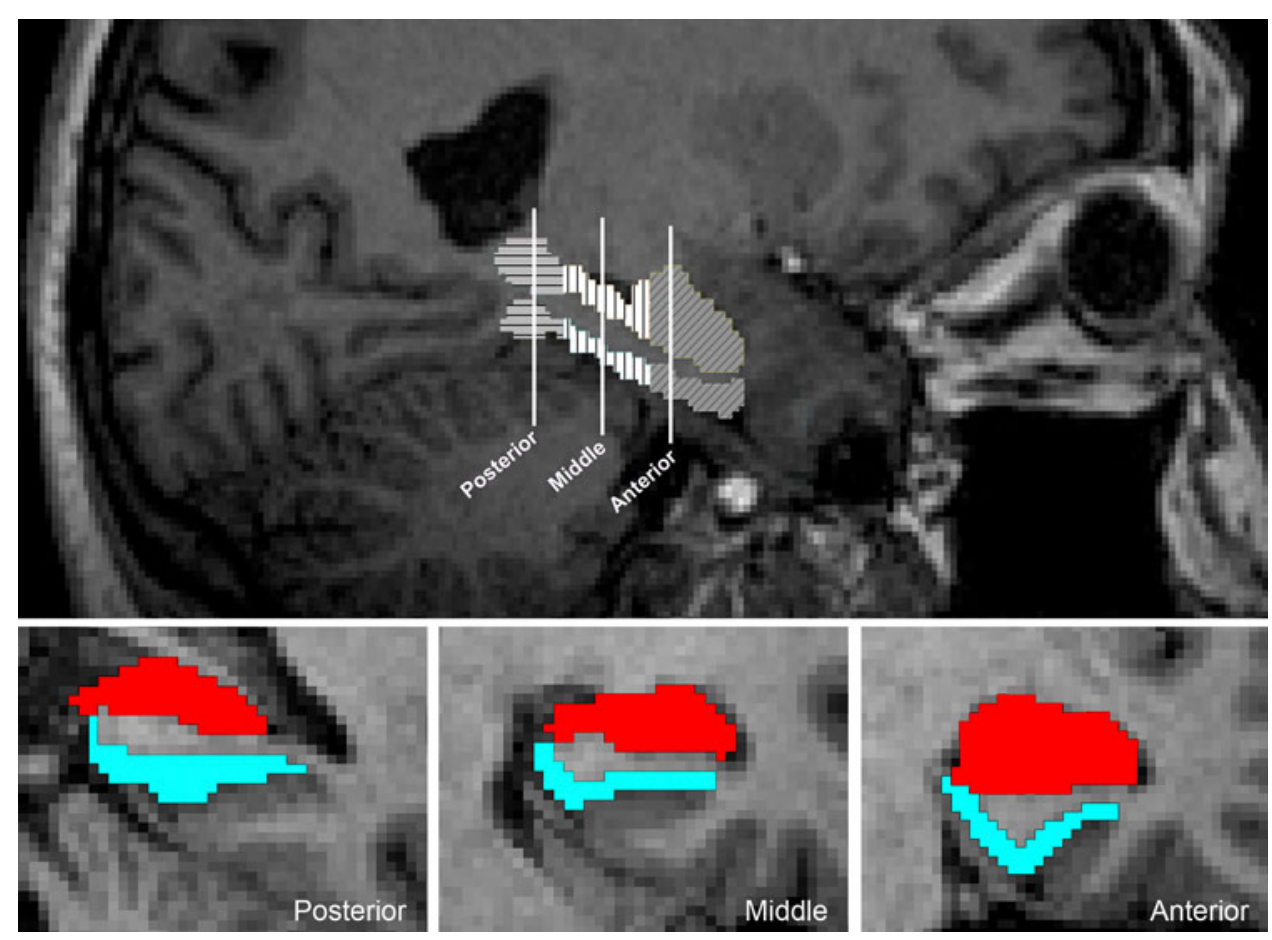
stage (MMSE score 10 and 15). The mean educational level in the control group was 4.2 (SD 1.4). The mean educational level in the MCI and the AD group was 3.8 (SD 1.8).

Volumetric comparisons between the three groups are summarized in Tables 1, 2. Age and intracranial volume were included as covariates to correct for possible age and cohort effects. With regard to the main effect of group on the total volumes of the hippocampus and parahippocampal gyrus: differences between the three groups were significant in both volumes (parahippocampal gyrus: $\mathrm{F}(2,46)=$ 24.23, $p<0.001$, hippocampus: $\mathrm{F}(2,46)=12.07, p<$ 0.001 ). Volumes were significantly different in the following order: healthy $>\mathrm{aMCI}>\mathrm{AD}$. ANCOVA analyses demonstrated that the parahippocampal gyrus is better in discriminating between groups than the hippocampal gyrus. When we added the hippocampus as covariate in the ANCOVA analysis with the parahippocampal gyrus as dependent variable, we still found robust significant group differences $(\mathrm{F}(2,45)=11.07 ; p<0.001)$. In contrast, when we added the parahippocampal gyrus as covariate in the ANCOVA analysis with the hippocampus as dependent variable, the significant group differences disappeared $(\mathrm{F}(2,45)=2.42 ; p=0.100)$. This indicates that the parahippocampal volume has added value on top of the discriminating ability of the hippocampus. Furthermore, pairwise comparisons between the healthy and aMCI groups showed a significant difference in the parahippocampal gyrus $(12.4 \%, \mathrm{~F}(1,29)=10.46, p=0.003)$, but not in the hippocampus $(1.7 \%, \mathrm{~F}(1,29)=0.85, p=0.362)$. When we compared the control or the aMCI group with the $\mathrm{AD}$ group, significant differences were found in both structures (Fig. 2).

With respect to the differences along the longitudinal axis in the hippocampus and parahippocampal gyrus, the repeated-measures GLM showed a significant group by structure interaction $(p=0.034)$ in the left parahippocampal gyrus when we compared controls with $\mathrm{AD}$ patients. The group difference was larger in the anterior part than in the middle and posterior parts. No other

Table 1 Volumetric comparisons between the three groups

\begin{tabular}{|c|c|c|c|c|c|c|}
\hline Volume in $\mathrm{mm}^{3}$ & $\begin{array}{l}\text { Controls }(n=18) \\
\text { Mean }(S D)\end{array}$ & $\begin{array}{l}\text { aMCI }(n=18) \\
\text { Mean }(\mathrm{SD})\end{array}$ & $\begin{array}{l}\mathrm{AD}(n=18) \\
\text { Mean }(\mathrm{SD})\end{array}$ & $\mathrm{F}(2,46)$ & $r$ & $p$ \\
\hline HCG total & 7464 (794) & 7334 (111) & $5922(122)$ & 12.0 & 0.1 & 0.000 \\
\hline $\mathrm{HCG}$ ant & 2959 (375) & $2970(459)$ & $2452(500)$ & 8.4 & 0.4 & 0.001 \\
\hline HCG middle & 2434 (269) & $2321(333)$ & 1915 (394) & 10.0 & 0.5 & 0.000 \\
\hline HCG post & $2100(359)$ & $2045(434)$ & $1592(383)$ & 7.0 & 0.4 & 0.002 \\
\hline PHG total & $4623(680)$ & 4047 (732) & $3014(588)$ & 24.2 & 0.7 & 0.000 \\
\hline PHG ant & 1657 (318) & $1389(326)$ & $954(303)$ & 16.6 & 0.6 & 0.000 \\
\hline PHG middle & $1646(322)$ & $1482(421)$ & $1074(298)$ & 15.6 & 0.6 & 0.000 \\
\hline PHG post & $1.335(202)$ & 1169 (170) & $881(200)$ & 20.3 & 0.6 & 0.000 \\
\hline HCG total L & 3773 (344) & $3704(450)$ & $3050(684)$ & 11.6 & 0.5 & 0.000 \\
\hline HCG ant L & 1503 (210) & 1500 (187) & $1241(156)$ & 7.4 & 0.4 & 0.002 \\
\hline HCG middle L & 1239 (154) & 1194 (154) & 994 (217) & 11.3 & 0.5 & 0.000 \\
\hline HCG post L & $1031(207)$ & 1009 (188) & $813(241)$ & 4.1 & 0.4 & 0.023 \\
\hline HCG total R & 3719 (575) & $3630(709)$ & $2872(620)$ & 9.4 & 0.4 & 0.000 \\
\hline HCG ant R & 1455 (276) & $1470(272)$ & 1204 (287) & 5.8 & 0.3 & 0.005 \\
\hline HCG middle $\mathrm{R}$ & 1194 (183) & 1123 (216) & $920(198)$ & 11.3 & 0.4 & 0.000 \\
\hline HCG post R & 1069 (159) & $1036(231)$ & 747 (214) & 6.8 & 0.4 & 0.000 \\
\hline PHG total L & $2321(487)$ & $2111(374)$ & 1468 (319) & 18.3 & 0.6 & 0.000 \\
\hline PHG ant L & 843 (212) & 705 (203) & 436 (184) & 11.7 & 0.6 & 0.000 \\
\hline PHG middle L & 839 (205) & $188(794)$ & 555 (129) & 12.7 & 0.5 & 0.000 \\
\hline PHG post $\mathrm{L}$ & 637 (142) & $611(106)$ & $476(123)$ & 8.3 & 0.5 & 0.001 \\
\hline PHG total R & 2301 (274) & $1936(429)$ & $1546(329)$ & 18.9 & 0.6 & 0.000 \\
\hline PHG ant $\mathrm{R}$ & $802(162)$ & $683(208)$ & $518(177)$ & 8.3 & 0.5 & 0.001 \\
\hline PHG middle $\mathrm{R}$ & $812(100)$ & 693 (159) & $586(140)$ & 11.1 & 0.6 & 0.000 \\
\hline PHG post $\mathrm{R}$ & 687 (130) & $558(122)$ & $441(115)$ & 15.3 & 0.6 & 0.000 \\
\hline
\end{tabular}

Note The group differences were calculated with ANCOVA (Univariate General Linear Model)

$H C G$ hippocampus, $P H G$ parahippocampal gyrus, ant anterior, post posterior, $L$ left, $R$ right, $S D$ standard deviation, $r$ effect size by Pearson's correlation coefficient, $p$ p value; $F(2,46)$ F-ratio and the degrees of freedom, $p, r$ and $F$ represent group difference 
Table 2 Volumetric pairwise comparisons

\begin{tabular}{|c|c|c|c|c|c|c|c|c|c|c|c|c|}
\hline \multirow[t]{2}{*}{ Volume in $\mathrm{mm}^{3}$} & \multicolumn{4}{|c|}{ Control and aMCI } & \multicolumn{4}{|c|}{$\mathrm{aMCI}$ and $\mathrm{AD}$} & \multicolumn{4}{|c|}{ Control and $\mathrm{AD}$} \\
\hline & $\%$ & $F(1,29)$ & $r$ & $p$ & $\%$ & $\mathrm{~F}(1,29)$ & $r$ & $p$ & $\%$ & $F(1,29)$ & $r$ & $p$ \\
\hline HCG total & 1.7 & 0.8 & 0.0 & 0.362 & 19.2 & 12.2 & 0.5 & 0.002 & 20.6 & 22.7 & 0.6 & 0.000 \\
\hline HCG ant & -0.3 & 0.2 & 0.0 & 0.628 & 17.8 & 9.2 & 0.5 & 0.005 & 17.1 & 14.4 & 0.5 & 0.001 \\
\hline HCG middle & 4.6 & 3.5 & 0.1 & 0.069 & 17.4 & 12.5 & 0.5 & 0.001 & 21.3 & 30.8 & 0.6 & 0.000 \\
\hline HCG post & 2.5 & 0.3 & 0.0 & 0.559 & 22.0 & 8.3 & 0.4 & 0.007 & 24.1 & 15.3 & 0.5 & 0.001 \\
\hline PHG total & 12.4 & 10.4 & 0.3 & 0.003 & 25.5 & 24.5 & 0.6 & 0.000 & 34.8 & 36.8 & 0.7 & 0.000 \\
\hline PHG ant & 16.1 & 9.4 & 0.3 & 0.004 & 31.3 & 12.8 & 0.5 & 0.001 & 42.4 & 25.2 & 0.7 & 0.000 \\
\hline PHG middle & 13.2 & 4.9 & 0.2 & 0.033 & 19.3 & 17.4 & 0.5 & 0.000 & 34.7 & 25.2 & 0.7 & 0.000 \\
\hline PHG post & 12.4 & 9.8 & 0.4 & 0.004 & 24.6 & 21.6 & 0.6 & 0.000 & 34.0 & 27.2 & 0.7 & 0.000 \\
\hline HCG total L & 1.8 & 1.2 & 0.0 & 0.266 & 17.6 & 10.9 & 0.5 & 0.003 & 19.1 & 19.8 & 0.5 & 0.000 \\
\hline $\mathrm{HCG}$ ant $\mathrm{L}$ & 0.19 & 0.4 & 0.0 & 0.499 & 17.2 & 8.0 & 0.4 & 0.008 & 17.4 & 11.6 & 0.5 & 0.002 \\
\hline HCG middle L & 3.6 & 1.4 & 0.1 & 0.233 & 16.7 & 12.7 & 0.5 & 0.001 & 19.7 & 19.7 & 0.5 & 0.000 \\
\hline HCG post $\mathrm{L}$ & 2.1 & 0.2 & 0.0 & 0.654 & 19.4 & 5.0 & 0.4 & 0.032 & 21.1 & 7.6 & 0.4 & 0.010 \\
\hline HCG total R & 2.3 & 0.7 & 0.0 & 0.379 & 20.8 & 10.0 & 0.4 & 0.004 & 22.7 & 19.7 & 0.5 & 0.000 \\
\hline HCG ant R & -1.0 & 0.0 & 0.0 & 0.844 & 18.9 & 7.7 & 0.4 & 0.009 & 17.2 & 10.4 & 0.4 & 0.003 \\
\hline HCG middle $\mathrm{R}$ & 5.9 & 3.7 & 0.1 & 0.063 & 18.0 & 8.1 & 0.4 & 0.008 & 22.9 & 24.4 & 0.5 & 0.000 \\
\hline HCG post $\mathrm{R}$ & 3.08 & 0.3 & 0.0 & 0.580 & 27.7 & 8.5 & 0.4 & 0.007 & 30.1 & 14.1 & 0.5 & 0.001 \\
\hline PHG total L & 9.04 & 3.4 & 0.2 & 0.071 & 30.4 & 36.9 & 0.7 & 0.000 & 36.7 & 24.4 & 0.7 & 0.000 \\
\hline PHG ant L & 16.3 & 4.6 & 0.3 & 0.039 & 38.1 & 10.9 & 0.5 & 0.003 & 48.2 & 19.9 & 0.7 & 0.000 \\
\hline PHG middle L & 17.9 & 1.1 & 0.1 & 0.282 & 19.3 & 26.8 & 0.6 & 0.000 & 33.8 & 17.7 & 0.6 & 0.000 \\
\hline PHG post L & 4.08 & 0.8 & 0.1 & 0.368 & 22.0 & 15.6 & 0.5 & 0.000 & 25.3 & 10.4 & 0.5 & 0.003 \\
\hline PHG total R & 15.8 & 18.4 & 0.4 & 0.000 & 20.1 & 8.0 & 0.4 & 0.008 & 32.8 & 35.4 & 0.7 & 0.000 \\
\hline PHG ant R & 20.4 & 6.4 & 0.3 & 0.016 & 18.8 & 4.5 & 0.4 & 0.042 & 35.4 & 13.9 & 0.6 & 0.000 \\
\hline PHG middle $\mathrm{R}$ & 14.6 & 10.2 & 0.4 & 0.003 & 15.4 & 4.1 & 0.4 & 0.050 & 27.8 & 21.1 & 0.7 & 0.000 \\
\hline PHG post $\mathrm{R}$ & 19.02 & 11.3 & 0.4 & 0.002 & 20.9 & 7.7 & 0.4 & 0.009 & 35.8 & 23.7 & 0.7 & 0.000 \\
\hline
\end{tabular}

Note The group differences were calculated with ANCOVA (Univariate General Linear Model)

$H C G$ hippocampus, $P H G$ parahippocampal gyrus; \% difference between the two groups (V1-V2 $\times 100 / \mathrm{V} 1), r$ effect size by Pearson's correlation coefficient, $p$ p value, $F(d f)$ for the covariate effect we give the F-ratio and the degrees of freedom (df)

significant differences were found between anterior, middle, and posterior parts. Furthermore, repeated-measures analysis showed no significant differences between both hemispheres (i.e., left-right differences).

\section{Discussion}

The main aim of the present study was to compare the volume differences in the hippocampus and parahippocampal gyrus between healthy controls, individuals with aMCI, and patients with AD. In addition, differences along the longitudinal axis of both structures were investigated. Our results suggest that parahippocampal volume discriminates better than hippocampal volume especially in the early phase of AD. No significant differences were found along the longitudinal axis, except for the left parahippocampal gyrus in which the anterior parts showed significantly larger differences than the posterior parts when controls were compared to AD patients.
Our finding that parahippocampal volume discriminates better than hippocampal volume is in line with the findings of most previous studies on pathological aging in which a comparison was made between healthy and MCI groups (Detoledo-Morrell et al. 2000; Jack et al. 2000; Jauhiainen et al. 2009; Killiany et al. 2002; Pennanen et al. 2004; Xu et al. 2000), between MCI and AD groups (Du et al. 2001; Jauhiainen et al. 2009; Tapiola et al. 2008; Visser et al. 1999; Xu et al. 2000), or between healthy and AD groups (Detoledo-Morrell et al. 2000; Dickerson et al. 2001; Du et al. 2001; Jauhiainen et al. 2009; Killiany et al. 2002; Pennanen et al. 2004). There have been, however, also a few studies that found larger group differences in the hippocampus (Detoledo-Morrell et al. 1997; Jack et al. 1997; Pennanen et al. 2004; Visser et al. 2002). Nevertheless, in these cases a group of AD patients was always involved. Thus, in those cases where the hippocampus was found to be a better discriminator than the parahippocampal gyrus, the group comparisons were always between MCI and $\mathrm{AD}$, or between control and $\mathrm{AD}$, but never 
Fig. 2 Boxplot of the hippocampal and parahippocampal gyrus volumes in $\mathrm{mm}^{3}$ in controls, MCI and AD patients. **significant difference with control group; *significant difference with MCI group
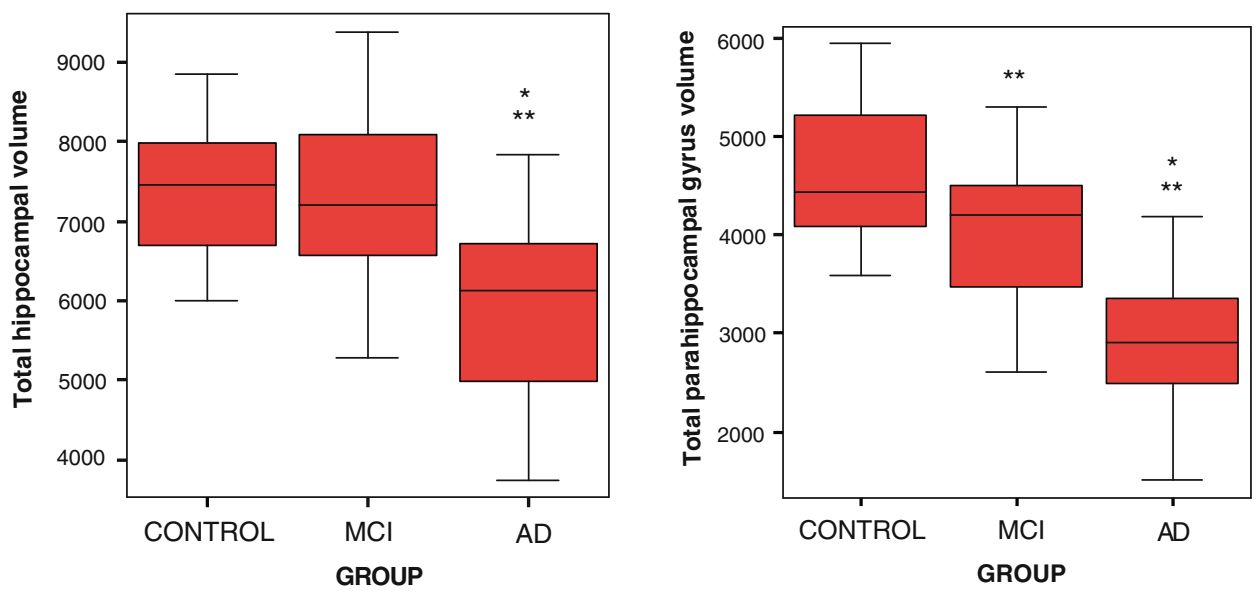

between control and MCI. This might indicate that the parahippocampal gyrus is a better discriminator particularly in the early (preclinical) phase of AD. The suggestion that the parahippocampal gyrus, in particular, is a highly sensitive marker with which to detect $\mathrm{AD}$ at a very early stage is in line with earlier reports on the entorhinal cortex (Detoledo-Morrell et al. 2004; Dickerson et al. 2001; Pennanen et al. 2004). The present study adds a new finding as it shows that this is not only the case with regard to the anterior part, but also with regard to the posterior part of the parahippocampal gyrus.

The present data are also partly in line with a previous study by our group (Burgmans et al. 2009) in which healthy, aging individuals with and without memory decline were compared. In that study, we also reported larger group differences in the parahippocampal gyrus. However, we found the largest group difference in the posterior part of the parahippocampal gyrus. This is in contrast with the present results, since here we found larger differences in the anterior part of the left parahippocampal gyrus than in the posterior parts of the left parahippocampal gyrus between controls and AD patients. A possible explanation for these different findings could be that atrophy in the posterior medial temporal lobe is more strongly related to healthy aging, whereas atrophy in the anterior medial temporal lobe is more strongly related to pathological aging. In a recent study, Raji et al. (2009) observed that in normal aging the most affected region was the posterior part of the hippocampus, while in $\mathrm{AD}$ patients the most affected regions were the anterior parts of the hippocampus and the parahippocampal gyrus.

There remain a few methodological issues to be addressed. First, our MRI data were acquired at only one time point, which precludes the assessment of actual pathological aging changes in volume, or of causality. Second, we included only male participants in order to decrease interindividual variation. We must, therefore, be cautious with generalizing our findings to females. Previous studies, however, did not reveal substantial genderrelated differences with respect to hippocampal and parahippocampal atrophy in AD. Third, the subdivision applied to the hippocampus and the parahippocampal gyrus was an MRI macroscopical one. We chose to divide both structures into three subregions along the longitudinal axis, because the literature suggests there are important differences in age-related and pathological-related changes between the anterior and posterior areas. However, there are no clear anatomical reasons for the cutoff points used in the present study (i.e., $35 \%$ for the anterior volume; $35 \%$ for the middle volume; and $30 \%$ for the posterior volume). Nevertheless, one of the aims of this study was to explore the group differences along the longitudinal axis, which has been made possible by our MRI-based approach. When it comes to investigating the subregions of the medial temporal lobe in more detail, cytoarchitectonic studies are considered more ideal.

In sum, our results suggest that parahippocampal volume discriminates better than hippocampal volume between cases of healthy aging, aMCI, and $\mathrm{AD}$, in particular, in the early phase of the disease. Our findings stress the importance of parahippocampal atrophy as an early biomarker of AD. Such predictive biological markers could be of great help for the development of early interventions designed to retard the progression of the disease. A possible implication could be a visual rating scale of the parahippocampal gyrus, similar to the one that exists for the hippocampus (Scheltens et al., 1992), which could help detect the earliest neurodegenerative changes indicative of $\mathrm{AD}$ in routine clinical practice.

Open Access This article is distributed under the terms of the Creative Commons Attribution Noncommercial License which permits any noncommercial use, distribution, and reproduction in any medium, provided the original author(s) and source are credited. 


\section{References}

Braak H, Braak E (1990) Neurofibrillary changes confined to the entorhinal region and an abundance of cortical amyloid in cases of presenile and senile dementia. Acta Neuropathol 80:479-486

Burgmans S, van Boxtel MP, van den Berg KE, Gronenschild EH, Jacobs HI, Jolles J, Uylings HB (2009) The posterior parahippocampal gyrus is preferentially affected in age-related memory decline. Neurobiol Aging. doi:10.1016/j.neurobiolaging.2009. 09.008

Detoledo-Morrell L, Sullivan MP, Morrell F, Wilson RS, Bennett DA, Spencer S (1997) Alzheimer's disease: in vivo detection of differential vulnerability of brain regions. Neurobiol Aging 18:463-468

Detoledo-Morrell L, Goncharova I, Dickerson B, Wilson RS, Bennett DA (2000) From healthy aging to early Alzheimer's disease: in vivo detection of entorhinal cortex atrophy. Ann N Y Acad Sci 911:240-253

DeToledo-Morrell L, Stoub TR, Bulgakova M et al (2004) MRIderived entorhinal volume is a good predictor of conversion from MCI to AD. Neurobiol Aging 25:1197-1203

Dickerson BC, Goncharova I, Sullivan MP et al (2001) MRI-derived entorhinal and hippocampal atrophy in incipient and very mild Alzheimer's disease. Neurobiol Aging 22:747-754

Du AT, Schuff N, Amend D et al (2001) Magnetic resonance imaging of the entorhinal cortex and hippocampus in mild cognitive impairment and Alzheimer's disease. J Neurol Neurosurg Psychiatry 71:441-447

Folstein MF, Folstein SE, McHugh PR (1975) "Mini-mental state". A practical method for grading the cognitive state of patients for the clinician. J Psychiatr Res 12:189-198

Gronenschild EH, Burgmans S, Smeets F, Vuurman EF, Uylings HBM, Jolles J (2010) A time-saving and facilitating approach for segmentation of anatomically defined cortical regions: MRI volumetry. Psychiatry Res 181:211-218

Hackert VH, den Heijer T, Oudkerk M, Koudstaal PJ, Hofman A, Breteler MM (2002) Hippocampal head size associated with verbal memory performance in nondemented elderly. Neuroimage $17: 1365-1372$

Insausti R, Amaral DG (2008) Entorhinal cortex of the monkey: IV. Topographical and laminar organization of cortical afferents. J Comp Neurol 509:608-641

Insausti R, Insausti AM, Sobreviela MT, Salinas A, Martinez-Penuela JM (1998) Human medial temporal lobe in aging: anatomical basis of memory preservation. Microsc Res Tech 43:8-15

Jack CR Jr, Petersen RC, Xu YC et al (1997) Medial temporal atrophy on MRI in normal aging and very mild Alzheimer's disease. Neurology 49:786-794

Jack CR Jr, Petersen RC, Xu Y et al (2000) Rates of hippocampal atrophy correlate with change in clinical status in aging and AD. Neurology 55:484-489

Jack CR Jr, Knopman DS, Jagust WJ et al (2010) Hypothetical model of dynamic biomarkers of the Alzheimer's pathological cascade. Lancet Neurol 9:119-128

Jauhiainen AM, Pihlajamaki M, Tervo S et al (2009) Discriminating accuracy of medial temporal lobe volumetry and fMRI in mild cognitive impairment. Hippocampus 19:166-175

Killiany RJ, Hyman BT, Gomez-Isla T et al (2002) MRI measures of entorhinal cortex vs hippocampus in preclinical AD. Neurology 58:1188-1196

Laakso MP, Frisoni GB, Kononen M et al (2000) Hippocampus and entorhinal cortex in frontotemporal dementia and Alzheimer's disease: a morphometric MRI study. Biol Psychiatry 47:10561063
McKhann G, Drachman D, Folstein M, Katzman R, Price D, Stadlan EM (1984) Clinical diagnosis of Alzheimer's disease: report of the NINCDS-ADRDA Work Group under the auspices of Department of Health and Human Services Task Force on Alzheimer's Disease. Neurology 34:939-944

Pennanen C, Kivipelto M, Tuomainen S et al (2004) Hippocampus and entorhinal cortex in mild cognitive impairment and early AD. Neurobiol Aging 25:303-310

Petersen RC, Smith GE, Waring SC, Ivnik RJ, Tangalos EG, Kokmen E (1999) Mild cognitive impairment: clinical characterization and outcome. Arch Neurol 56:303-308

Petersen RC, Doody R, Kurz A et al (2001) Current concepts in mild cognitive impairment. Arch Neurol 58:1985-1992

Raji CA, Lopez OL, Kuller LH, Carmichael OT, Becker JT (2009) Age, Alzheimer disease, and brain structure. Neurology 73:1899-1905

Raz N, Lindenberger U, Rodrigue KM et al (2005) Regional brain changes in aging healthy adults: general trends, individual differences and modifiers. Cereb Cortex 15:1676-1689

Scheltens P, Leys D, Barkhof F et al (1992) Atrophy of medial temporal lobes on MRI in "probable" Alzheimer's disease and normal ageing: diagnostic value and neuropsychological correlates. J Neurol Neurosurg Psychiatry 55:967-972

Scher AI, Xu Y, Korf ES et al (2007) Hippocampal shape analysis in Alzheimer's disease: a population-based study. Neuroimage 36:8-18

Shrout PE, Fleiss JL (1979) Intraclass correlations: uses in assessing rater reliability. Psychol Bull 86:420-428

Smith SM (2002) Fast robust automated brain extraction. Hum Brain Mapp 17:143-155

Tapiola T, Pennanen C, Tapiola M et al (2008) MRI of hippocampus and entorhinal cortex in mild cognitive impairment: a follow-up study. Neurobiol Aging 29:31-38

Thangavel R, Van Hoesen GW, Zaheer A (2008) Posterior parahippocampal gyrus pathology in Alzheimer's disease. Neuroscience 154:667-676

Van de Pol LA, Hensel A, van der Flier WM et al (2006) Hippocampal atrophy on MRI in frontotemporal lobar degeneration and Alzheimer's disease. J Neurol Neurosurg Psychiatry 77:439-442

Van der Elst W, van Boxtel MP, van Breukelen GJ, Jolles J (2005) Rey's verbal learning test: normative data for 1855 healthy participants aged 24-81 years and the influence of age, sex, education, and mode of presentation. $\mathrm{J}$ Int Neuropsychol Soc 11:290-302

Van Hoesen GW (1982) The parahippocampal gyrus: new observations regarding its cortical connections in the monkey. Trends in neurosciences 5

Visser PJ, Scheltens P, Verhey FR et al (1999) Medial temporal lobe atrophy and memory dysfunction as predictors for dementia in subjects with mild cognitive impairment. J Neurol 246:477-485

Visser PJ, Verhey FR, Hofman PA, Scheltens P, Jolles J (2002) Medial temporal lobe atrophy predicts Alzheimer's disease in patients with minor cognitive impairment. J Neurol Neurosurg Psychiatry 72:491-497

Wang L, Miller JP, Gado MH et al (2006) Abnormalities of hippocampal surface structure in very mild dementia of the Alzheimer type. Neuroimage 30:52-60

Weniger G, Irle E (2006) Posterior parahippocampal gyrus lesions in the human impair egocentric learning in a virtual environment. Eur J Neurosci 24:2406-2414

$\mathrm{Xu}$ Y, Jack CR Jr, O'Brien PC et al (2000) Usefulness of MRI measures of entorhinal cortex versus hippocampus in $\mathrm{AD}$. Neurology 54:1760-1767 